\title{
Improved quality of auditory event-related potentials recorded simultaneously with 3-T fMRI: removal of the ballistocardiogram artefact
}

Citation for published version (APA):

Debener, S., Strobel, A., Sorger, B., Peters, J., Kranczioch, C., Engel, A. K., \& Goebel, R. (2007).

Improved quality of auditory event-related potentials recorded simultaneously with 3-T fMRI: removal of the ballistocardiogram artefact. Neuroimage, 34(2), 587-597.

https://doi.org/10.1016/j.neuroimage.2006.09.031

Document status and date:

Published: 01/01/2007

DOI:

10.1016/j.neuroimage.2006.09.031

Document Version:

Publisher's PDF, also known as Version of record

Document license:

Taverne

Please check the document version of this publication:

- A submitted manuscript is the version of the article upon submission and before peer-review. There can be important differences between the submitted version and the official published version of record.

People interested in the research are advised to contact the author for the final version of the publication, or visit the DOI to the publisher's website.

- The final author version and the galley proof are versions of the publication after peer review.

- The final published version features the final layout of the paper including the volume, issue and page numbers.

Link to publication

\footnotetext{
General rights rights.

- You may freely distribute the URL identifying the publication in the public portal. please follow below link for the End User Agreement:

www.umlib.nl/taverne-license

Take down policy

If you believe that this document breaches copyright please contact us at:

repository@maastrichtuniversity.nl

providing details and we will investigate your claim.
}

Copyright and moral rights for the publications made accessible in the public portal are retained by the authors and/or other copyright owners and it is a condition of accessing publications that users recognise and abide by the legal requirements associated with these

- Users may download and print one copy of any publication from the public portal for the purpose of private study or research.

- You may not further distribute the material or use it for any profit-making activity or commercial gain

If the publication is distributed under the terms of Article $25 \mathrm{fa}$ of the Dutch Copyright Act, indicated by the "Taverne" license above, 


\title{
Improved quality of auditory event-related potentials recorded simultaneously with 3-T fMRI: Removal of the ballistocardiogram artefact
}

\author{
Stefan Debener, ${ }^{\mathrm{a}, \mathrm{b}, *}$ Alexander Strobel, ${ }^{\mathrm{c}, \mathrm{d}, \mathrm{e}}$ Bettina Sorger, ${ }^{\mathrm{d}, \mathrm{e}}$ Judith Peters, ${ }^{\mathrm{d}, \mathrm{e}}$ \\ Cornelia Kranczioch, ${ }^{f, g}$ Andreas K. Engel, ${ }^{f}$ and Rainer Goebel ${ }^{\mathrm{d}, \mathrm{e}}$ \\ ${ }^{\mathrm{a}}$ MRC Institute of Hearing Research Southampton, Royal South Hants Hospital, Southampton, UK \\ ${ }^{\mathrm{b}}$ School of Medicine, University of Southampton, Southampton, UK \\ ' Institute of Psychology, University of Technology Dresden, Dresden, Germany \\ ${ }^{\mathrm{d} D e p a r t m e n t ~ o f ~ N e u r o c o g n i t i o n, ~ F a c u l t y ~ o f ~ P s y c h o l o g y, ~ M a a s t r i c h t ~ U n i v e r s i t y, ~ T h e ~ N e t h e r l a n d s ~}$ \\ e M-BIC Maastricht Brain Imaging Center, Maastricht, The Netherlands \\ ${ }^{\mathrm{f}}$ Department of Neurophysiology and Pathophysiology, University Medical Center Eppendorf, Hamburg, Germany \\ ${ }^{\mathrm{g}}$ University of Portsmouth, Portsmouth, UK
}

Received 17 April 2006; revised 15 September 2006; accepted 22 September 2006

EEG signals recorded simultaneously with fMRI are massively compromised by severe artefacts, among them the cardiac cyclerelated ballistocardiogram (BCG) artefact. Different methods have been proposed to remove the BCG artefact focusing on channel-wise template subtraction procedures or spatial filtering approaches such as independent component analysis (ICA). Here we systematically compared the performance of the optimal basis set (OBS), a channel-wise correction approach, with ICA and a recently proposed combination of both (OBS-ICA). The three different procedures were applied to 60-channel EEG data from 12 subjects recorded during fMRI acquisition in a 3-T scanner. In addition to examination of the residual BCG artefact, the signal-to-noise ratio (SNR) and the topography of the resulting auditory evoked potential component N1 were compared. Whereas all three approaches led to a significant artefact reduction, the ICA procedure resulted in a significantly reduced N1 SNR and amplitude when compared to BCG-uncorrected data, indicating a rather poor performance. In contrast to ICA, OBS and OBS-ICA corrected data substantially improved the SNR of the N1. The quality of the auditory evoked potential N1 topography was investigated by means of equivalent current dipole modelling. On a descriptive level, all three correction procedures led to a reduced localization error when compared to BCG-uncorrected data. This improvement was significant for OBS-ICA. We conclude that OBS and OBS-ICA can efficiently remove BCG artefacts and substantially improve the quality of EEG signals recorded inside the scanner, a

\footnotetext{
* Corresponding author. MRC Institute of Hearing Research, Royal South Hants Hospital, Southampton SO14 OYG, UK. Fax: +44 2380825611. E-mail address: sdebener@soton.ac.uk (S. Debener).

Available online on ScienceDirect (www.sciencedirect.com).
}

prerequisite for the successful integration of simultaneously recorded EEG and fMRI.

(C) 2006 Elsevier Inc. All rights reserved.

Keywords: Simultaneous EEG/fMRI; Independent component analysis; Ballistocardiogram artefact; Dipole modelling; Auditory evoked potential N1

Introduction

Electroencephalography (EEG) and functional magnetic resonance imaging (fMRI) provide complimentary information with regard to the spatial and temporal resolution of brain activity. Accordingly, the combined recording and analysis of both signals holds great promise for a better understanding of brain function and dysfunction (Kruggel et al., 2000). However, the EEG signal recorded during fMRI acquisition is heavily compromised by severe artefacts. A further concern for auditory research in particular is the acoustic noise generated by the scanner (Brechmann et al., 2002; Hall et al., 1999, 2000). Thus, the standard MR environment can be considered a worst case scenario for the recording of auditory evoked potentials (AEP). AEPs are therefore an excellent candidate for the examination of the quality of event-related potentials (ERPs) recorded simultaneously with fMRI. Here we investigate how different MR artefact correction procedures affect the quality of the AEP.

Apart from body movements, EEG data recorded inside the MR scanner are compromised by two major artefacts. The gradient artefact reflects the switching of magnetic fields during MRI image 
acquisition and can cause amplitudes orders of magnitudes larger than the EEG signal. One way to bypass this problem is to record EEG and fMRI data alternately. However, this approach limits the potential of simultaneous EEG/fMRI applications. Procedures therefore have been developed to correct the EEG signal from gradient artefacts. Since the gradient artefact is relatively invariant over time (Garreffa et al., 2004), it is possible to apply an average artefact template procedure and subtract it out (Allen et al., 2000).

Due to its variability and its contribution in the EEG and eventrelated potential (ERP) frequency range $(0.5-25 \mathrm{~Hz})$, the ballistocardiogram (BCG) artefact poses a greater challenge. The $\mathrm{BCG}$ artefact is related to the cardiac cycle and seems to represent a combination of the pulse-related movement of scalp electrodes and electrically conductive blood (Sijbers et al., 2000; Srivastava et al., 2005). Similar to gradient artefact correction, a powerful BCG correction approach is based on the channel-wise subtraction of a global average (Allen et al., 1998) or local average (Ellingson et al., 2004) artefact template. The focus on channel-wise instead of topographic, across-channel BCG correction is based on the observation that morphology, amplitude and peak latency of the BCG artefact are usually different between channels. However, given the considerable trial-by-trial variability of the BCG artefact, a single template-based correction procedure leaves space for further improvements. Therefore, an alternative method has been developed that is based on a temporal principal components analysis (PCA) on each channel and on constructing several artefact templates (Niazy et al., 2005). This method has been named optimal basis set (OBS) and does not assume the exact repetition of the same artefact shape. Compared to a single template approach, the OBS has been shown to further reduce the residual $\mathrm{BCG}$ artefact, indicating improved $\mathrm{BCG}$ correction performance (Niazy et al., 2005).

Other groups have followed a topographically oriented correction of BCG artefacts by means of PCA or independent component analysis (ICA) (Benar et al., 2003; Eichele et al., 2005; Srivastava et al., 2005). Here a set of channel-weights is defined, which, when matrix-multiplied with the data, returns orthogonal (PCA) or maximally temporally independent (ICA) component activations. It has been reported that up to six independent components (IC) jointly accounted for the BCG artefact (Benar et al., 2003; Eichele et al., 2005; Srivastava et al., 2005). Benar et al. (2003) compared PCA with ICA and showed that both methods performed well in eliminating BCG artefacts, with ICA producing a clearer separation from non-artefact signals.

For EEG data recorded outside the scanner, ICA is a powerful tool for the correction of EEG artefacts such as eye blinks and lateral eye movements (Jung et al., 2000a,b). Moreover, it has been shown that ICA helps to disentangle spatio-temporally overlapping event-related EEG signals (Delorme et al., 2002; Makeig et al., 2004a,b; Onton et al., 2005) and can help to identify selected ERP components on a single-trial level (Debener et al., 2005a). For EEG data recorded inside the scanner, we recently observed that the application of ICA after OBS-based BCG artefact correction substantially improved EEG quality (Debener et al., 2005b). However, as a systematic comparison is lacking, it is currently not known whether a channel-wise, spatial, or combined approach performs best for BCG artefact removal. In the present study, we therefore compared three different BCG correction procedures, namely OBS (Niazy et al., 2005), ICA (Benar et al., 2003; Eichele et al., 2005), and the combined OBS-ICA approach (Debener et al., 2005b). Note that each of these procedures could easily result in an overcorrection of the data. We therefore included a condition based on data uncorrected for the BCG artefact. For the same reason, the quality of the correction procedures was evaluated by comparing resulting AEPs in addition to the amount of residual BCG artefacts.

The quality of AEPs was determined by systematically comparing the N1 signal-to-noise ratio (SNR) and the quality of the N1 topography. Specifically, the auditory N1 SNR for BCGuncorrected data was compared to the auditory N1 SNR obtained from OBS, ICA, and OBS-ICA corrected data. For two reasons, the quality of the N1 topography was assessed by means of equivalent current dipole modelling. Firstly, the location of its main neuronal generator is known to be in the auditory cortex (Naatanen and Picton, 1987; Scarff et al., 2004; Scherg and Von Cramon, 1985). Although some discussion exists on the distinction of $\mathrm{N} 1$ subcomponents, the main contribution comes from supratemporal primary auditory cortex (Yvert et al., 2005). Accordingly, the most appropriate $\mathrm{BCG}$ correction procedure can be hypothesized to be the one providing a good N1 SNR and a source localization close to the primary auditory cortex. Secondly, equivalent current dipole modelling has been used to integrate ERP and fMRI information. This is usually done for data obtained from separate recording sessions (Bledowski et al., 2004, 2006; Heinze et al., 1994; Opitz et al., 1999; Schicke et al., 2006), but would profit from simultaneous EEG/fMRI recording protocols if ERPs of reasonable quality could be obtained.

\section{Methods}

Subjects

Participants were 16 healthy volunteers with no history of hearing disorders. Written informed consent was obtained prior to the experiment, in accordance with the local ethics committee guidelines. Data from four subjects had to be discarded due to technical malfunction (3) or poor data quality due to frequent body movements (1), leaving a final sample size of 12 participants (5 male, age range of 21-32 years).

\section{Task}

Stimuli were presented using Presentation 9 (Neurobehavioral Systems, San Francisco, CA). Subjects were presented with a variant of the three class auditory oddball task (Debener et al., 2002, 2003, 2005a). In alternating blocks, subjects attended to either rare target tones or environmental sounds, which were presented in pseudorandom order interspersed with frequent standard tones. In the present report we analysed ERPs in response to frequent standard trials only ( $80 \%$ probability). Two sinus tones ( $400 \mathrm{~ms}$ duration, $10 \mathrm{~ms}$ rise and fall time, 350 or $650 \mathrm{~Hz}$ ) served as frequent standards and were counterbalanced across subjects. Stimulus onset was timed between $1482 \mathrm{~ms}$ and $1591 \mathrm{~ms}$ after the onset of MR gradient switching, which lasted $1400 \mathrm{~ms}$. The mean inter-stimulus interval was $2000 \mathrm{~ms}$. A total of 920 standard trials were presented in 4 runs, with 1-min breaks in between, resulting in a recording duration of about $40 \mathrm{~min}$. The standard Siemens Allegra MR intercom system was used for stimulus delivery. In this system the sound pressure is delivered via tubes to passive headphones, which also served to reduce the scanner noise. The sound pressure level of the stimulus presentation was kept constant across subjects. After the experiment all subjects reported that they had heard the stimuli without difficulties. 


\section{fMRI and EEG recording}

Imaging was performed using a Siemens Allegra 3-T head scanner equipped with a standard head coil. Twenty-four functional slices were recorded parallel to the anterior commissure-posterior commissure line using a gradient echo planar imaging sequence with an echo time of $30 \mathrm{~ms}$, a flip angle of $90^{\circ}$, and a time to repeat (TR) of $2000 \mathrm{~ms}$. Similar to our previous study (Debener et al., $2005 \mathrm{~b}$ ), slice acquisition was timed such that a silent interval of $600 \mathrm{~ms}$ remained before the acquisition of the next volume. This was done for several reasons. Firstly, the procedure ensured that the EEG data quality could be visually monitored on all recording channels during data recording. Secondly, it is our experience that a silent interval helps reviewing the quality of offline MR gradient artifact removal. And thirdly, a silent interval was used to enable delivery of auditory stimuli during short periods not affected by scanner noise.

A 64-channel high-input impedance amplifier system specifically designed for the MR environment was used (Brainproducts, Munich, Germany). The setup consisted of two 32-channel MR plus amplifiers powered by a rechargeable power unit. The amplifiers were placed directly behind the scanner bore inside the MR room, which allowed the use of short wires with a total length of about $1.2 \mathrm{~m}$ from recording electrodes to amplifier. Sintered $\mathrm{Ag} / \mathrm{AgCl}$ ring electrodes with built-in $5 \mathrm{k} \Omega$ resistors were used. Data were recorded from 62 equidistant scalp sites mounted in a cap system (Easycap, Falk Minow Services, Herrsching, Germany). Additional plastic electrode holders were tied into the cap at occipital scalp sites which substantially improved subject comfort. Continuous data were also recorded from one electrode placed below the left eye to monitor eye blinks and another electrode placed at the lower back for electrocardiogram (ECG) recording. All 64-channel data were referenced to the vertex. The data were recorded with a passband of $0.016-250 \mathrm{~Hz}$ and digitized with 5000 samples/s at 16bit resolution, resulting in a dynamic range of $16.38 \mathrm{mV}$. The amplified signal was transmitted via fiber-optic cables to a recording PC placed outside the MR room. Electrode impedances were maintained below $20 \mathrm{k} \Omega$ before recordings.

\section{Removal of gradient artefact}

fMRI gradient artefacts were removed using a local average artefact template procedure (Allen et al., 2000) as implemented in Analyzer 1.05 (Brainproducts, Munich. Germany). Data were corrected relative to the MR volume gradient artefact onset, which was indicated by a trigger received from the MR system and recorded with the EEG. A moving average width of $30 \mathrm{MR}$ volumes (TRs) was used for gradient correction. Corrected EEG data were $0.3-40 \mathrm{~Hz}$ pass-band filtered, down-sampled to $250 \mathrm{~Hz}$, and exported to Matlab 7 (MathWorks, Natick, MA). Further processing was performed for 60 EEG channels, because after gradient artefact removal it became apparent that two channels placed at lower temporal sites, inferior to the usual 10-20 electrode space, provided poor EEG quality in several subjects.

\section{Rejection of artefacts}

Data processing under Matlab was based on EEGLAB 4.514 (Delorme and Makeig, 2004), an open source toolbox developed by the Swartz Center for Computational Neurosciences, La Jolla, CA (http://www.sccn.ucsd.edu/eeglab). The data were epoched from -200 to $800 \mathrm{~ms}$ relative to stimulus onset. Epochs containing non-stereotyped artefacts like gross body movements were excluded from further analysis using a rejection procedure as implemented in EEGLAB. By determining the probability distribution of the amplitude values across epochs, the probability of occurrence of each trial was computed for each channel and across all channels. Epochs with improbable amplitude values ( $>4$ standard deviations) were rejected from further analysis. This procedure ensured that the BCG artefact, which was present in every single trial, was left undetected, whereas rare artefact events such as eye blinks were reliably identified. On average, 853 valid epochs remained per subject (range 829 to 872 epochs). Time-domain averaging of these epochs resulted in AEPs based on BCG-uncorrected epochs. These data are further referred to as RAW-AEPs.

\section{Removal of ballistocardiogram artefact}

For the OBS-based BCG correction dataset, the EEGLAB plug-in FMRIB 1.2 (Niazy et al., 2005), as developed by the Centre for the Functional MRI of the Brain (Oxford, UK), was used (http://www.fmrib.ox.ac.uk/ rami/). The FMRIB plug-in provides a reliable heartbeat detection algorithm based on a Teager energy operator and adaptive thresholding. The heartbeat events are used to generate and subtract a BCG template from the continuous data. The continuous data are epoched relative to the heartbeat events and aligned in a matrix to calculate the first three principal components, which are then taken to form the OBS. The OBS are then least-squares-fitted and subtracted from each segment. Note that this procedure was performed separately for each channel. Time-domain averaging of these BCG-corrected epochs resulted in AEPs named OBS-AEPs.

For the ICA-based BCG correction, extended infomax ICA (Bell and Sejnowski, 1995; Lee et al., 1999) as implemented in EEGLAB was performed on the concatenated single-trial data matrix. A weight change of $10^{-7}$ as stop criterion resulted in a stable decomposition after less than 800 iterations. Matrix multiplication of the resulting square unmixing matrix with the raw data returned maximally independent time courses. Each IC can be characterized by its time course and a corresponding topography, given by the inverse of the unmixing weights. As proposed recently, ICs reflecting BCG artefact were identified by correlating their time courses with the recorded ECG signal (Srivastava et al., 2005). IC activations correlating with the ECG signal $(r>0.20)$ were considered to reflect BCG artefact. Furthermore, the topographies of these ICs were inspected for patterns similar to the BCG artefact topography. Across subjects, between two and four ICs were identified as BCG-related $($ mean $=3.3)$. Corrected EEG data were obtained by the joint back-projection of all remaining ICs. For these data, AEPs were computed and named ICA-AEPs.

For the combined OBS-ICA correction procedure (Debener et al., 2005b), extended infomax ICA was performed on the concatenated single-trial data following correction for BCG artefacts using the OBS method. Again, BCG-related IC activations were correlated with the ECG signal. However, in none of the subjects did any IC activation show a substantial correlation with the ECG signal $(r<0.10)$. Therefore, the IC reflecting residual $\mathrm{BCG}$ artefact was identified by correlating all 
IC topographies with the BCG artefact topography, the latter being determined at the global field power derived peak latency of the averaged BCG artefact. The IC with the highest correlation was considered to reflect residual BCG artefact and its contribution removed by joint back-projection of the remaining components. Following this approach, the mean correlation (after Fishers' $z$ transform) between BCG map and identified IC map across all subjects was $r=0.94$ (range, $r=0.81$ to 0.99 ). Visual inspection of the resulting components also confirmed the validity of this procedure. Time-domain averaging of the OBS-ICA corrected epochs resulted in a dataset named OBS-ICA AEPs.

\section{Residual BCG artefact}

For each of the four data sets (RAW, ICA, OBS, and OBSICA), the amount of residual BCG artefact was determined as follows. Data were epoched from $-100 \mathrm{~ms}$ to $400 \mathrm{~ms}$ relative to the onset of each heartbeat event, and then averaged. For the ECG channel this procedure returns the time-locked, evoked fraction of the ECG signal, and for the EEG channels it reflects the evoked BCG artefact, with the ongoing EEG being averaged out. The BCG artefact was quantified by taking the integral of the EEG channel envelope, which is given by the minima and maxima across all EEG channels. To test for significant differences regarding the
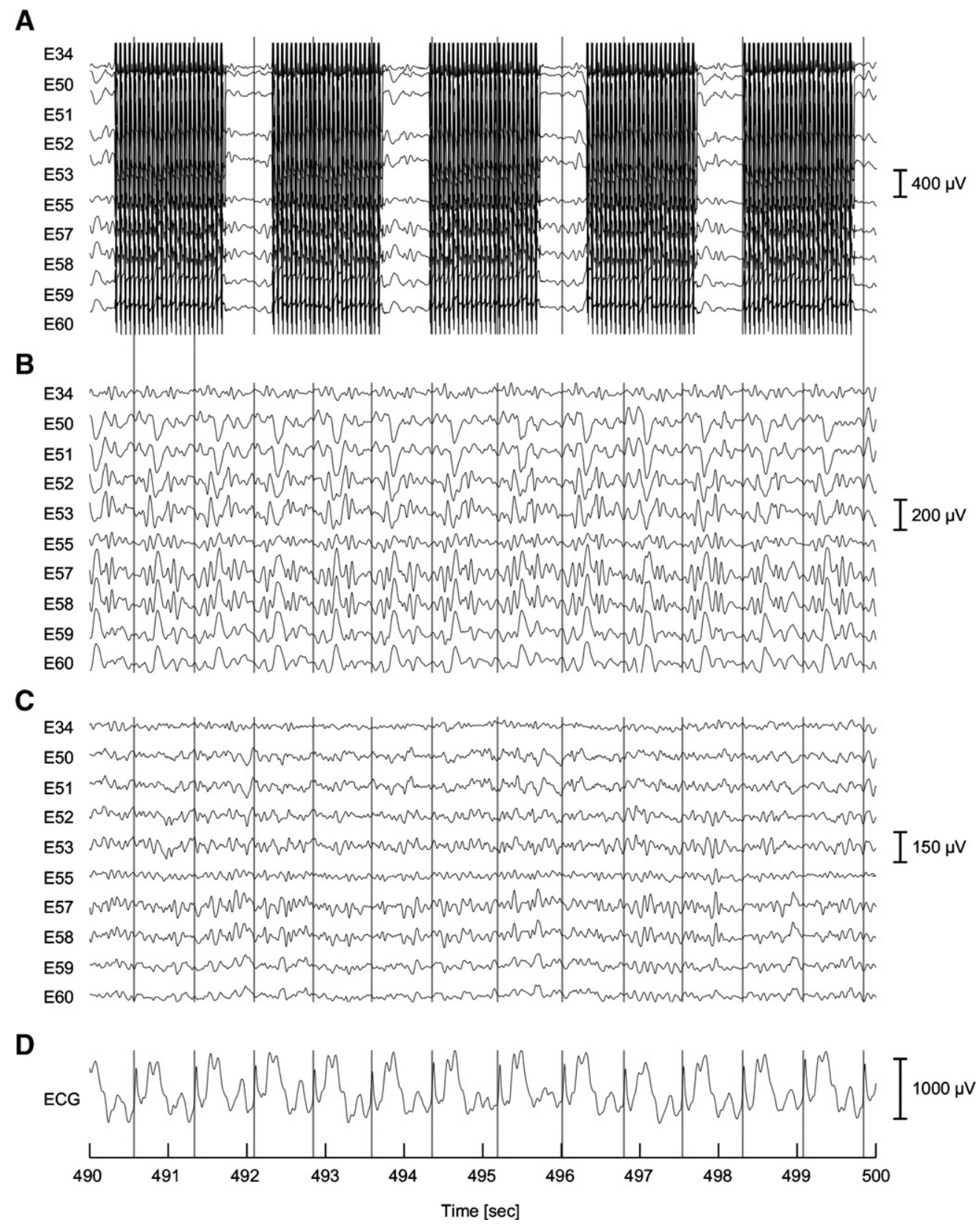

Fig. 1. 10-s example EEG data from a representative subject before (A) and after (B) gradient correction, and after removal of BCG artefacts using the OBS method (C). The corresponding ECG signal (D) is shown together with the identified heartbeat events (vertical grey lines). Note the different scaling between panels. For a topographical layout of the displayed 10 EEG channels, see the inset in Fig. 3. 
amount of the BCG artefact, a one-way ANOVA comprising all four data sets was computed. The Greenhouse-Geisser corrected $p$-value is reported and paired $t$-tests were used to follow-up the main effect.

\section{Signal-to-noise ratio of AEPS}

Note that for the four different datasets RAW, ICA, OBS, and OBS-ICA, the resulting AEPs were based on exactly the same epochs per subject. This ensured that AEP differences between data sets were solely due to the BCG correction procedure applied. AEPs were obtained after baseline correction of common average referenced data. The auditory $\mathrm{N} 1$ amplitude was identified as the maximum negative deflection at the fronto-central midline channel (E01) in the time interval $50 \mathrm{~ms}$ to $150 \mathrm{~ms}$. The SNR was computed by dividing the N1 peak amplitude by the standard deviation of the AEP in the pre-stimulus interval ( -200 to $0 \mathrm{~ms}$ ). An additional noise estimate was obtained for display purposes, by creating two sub-averages based on odd and even epoch indices. The difference between these two sub-averages indicates residual noise in ERPs (Schimmel, 1967). Since the SNR values were not normally distributed, the statistical significance between the four different datasets was assessed using non-parametric Wilcoxon tests.

\section{Equivalent current dipole modelling}

The quality of the resulting grand mean averaged AEP topographies was assessed by means of equivalent current dipole modelling, as implemented in BESA, Version 5.0.8 (MEGIS, Graefeling, Germany). A standard 4-shell ellipsoidal head model in Talairach space was used with default radii and conductivity parameters. The fit interval was set from $88 \mathrm{~ms}$ to $120 \mathrm{~ms}$, as indicated by the global field power derived onset-to-peak latency of the N1 (K. Hoechstetter and M. Scherg, personal communication). Two symmetric equivalent current dipoles were used to model the 1-20 Hz pass-band filtered data. For each of the datasets RAW, ICA, OBS, and OBS-ICA, the quality of the resulting fit was explored by computing the Euclidean distance between the fitted location and the reference location, which was defined as the mean coordinate of the Heschl gyrus (Talairach coordinates $[x, y, z]= \pm 49.5,-17,9)$. This reference location was derived by determining the extent of left and right Heschl's gyrus, which was based on a freely available software package (http:// www.bion.de) providing masks for region of interest analysis (Walter et al., 2003). The residual variance as determined by the source modelling procedure is also reported, and expresses the difference between the modelled and the measured scalp map. The free model and the reference model, i.e. two bilateral dipoles
A

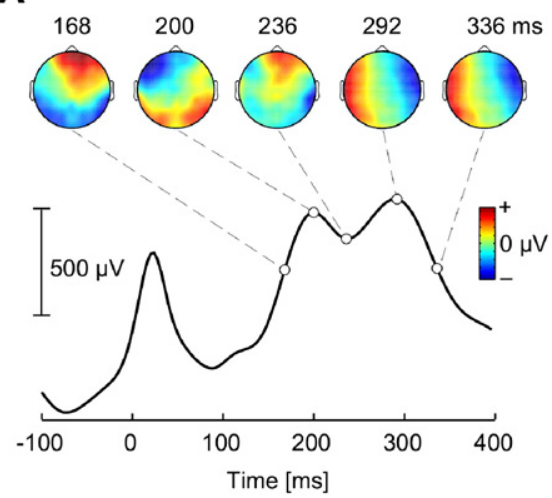

C

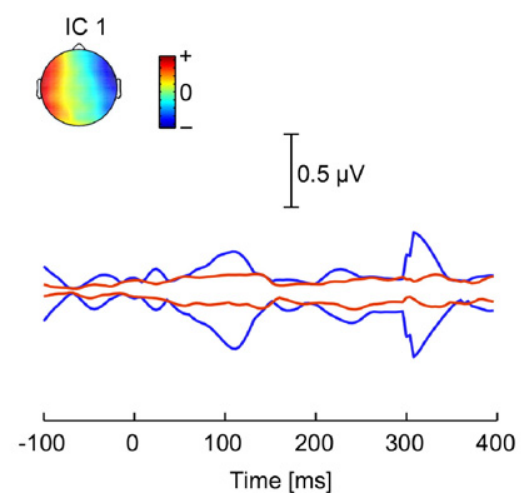

\section{B}

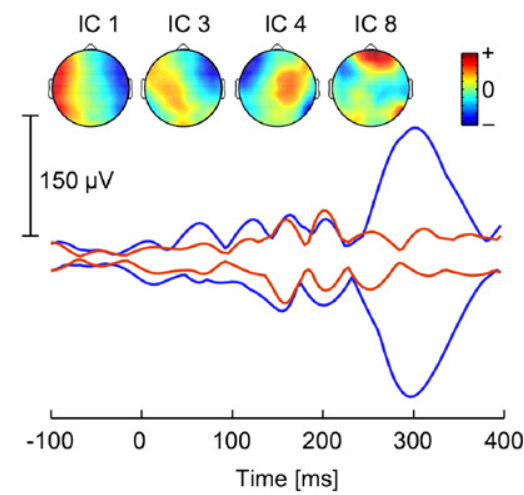

D

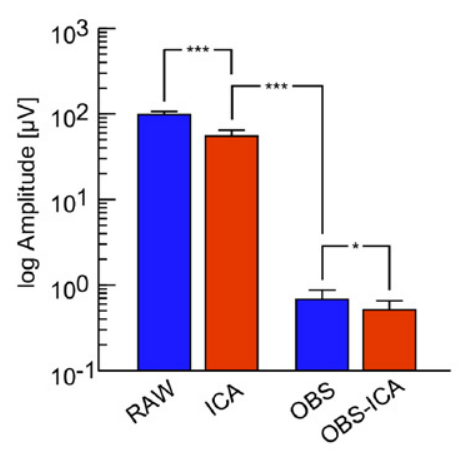

Fig. 2. Performance of different procedures to remove the BCG artefact. A single subject example of the averaged ECG signal (A) and corresponding scalp maps are shown at selected latencies. (B) The EEG channel envelope of the same subject before (blue) and after (red) correction using the ICA approach. The identified IC maps are displayed (arbitrary units, absmax scaled). (C) Same as in panel B for OBS-method (blue) and OBS-ICA approach (red). The identified IC representing residual artefact is displayed. Note the different scaling in panels B and C. (D) Mean BCG artefact amplitude across 12 subjects (+SEM) for the four different datasets. RAW = data uncorrected for the BCG artefact; ICA =ICA-based BCG correction; OBS = OBS-based BCG correction; OBS-ICA= combined approach, i.e., ICA-based BCG correction following OBS. ${ }^{* * *} p<0.0001 ;{ }^{*} p<0.05$. 
seeded into Heschl's gyrus, are reported. Source modelling of grand average AEP was complemented by applying the same reference model to single-subject AEP data and determining the localization error for each subject.

\section{Results}

A representative 10-s interval of ongoing EEG activity at ten lateral EEG channels is shown in Fig. 1. As can be seen, the massive gradient artefact during fMRI slice acquisition was absent after gradient correction. The quality of the gradient correction may be further estimated by inspection of the ECG channel (Fig. 1D), which is displayed after gradient correction only. The ECG morphology was apparently not disturbed by the gradient correction procedure. Fig. 1B also demonstrates the magnitude and variability of the BCG artefact at lateral channels. Note, for instance, the different morphology and time course of the BCG artefact between left (E57, E58, E59, E60) and right (E50, E51,
E52, E53) hemisphere channels. The major contributions of the BCG artefact followed each heartbeat cycle onset by about 150 to $400 \mathrm{~ms}$. As an example, the EEG data corrected for BCG artefacts using the OBS method are shown in Fig. 1C. A close inspection of the signals may suggest that residual BCG-related artefacts are still present, for instance at second 494 and 498. However, visual inspection of the ongoing EEG activity alone seems insufficient to determine the quality of the BCG correction procedure applied.

\section{Residual BCG artefact}

Fig. 2A shows the averaged ECG signal from the same subject as shown in Fig. 1. Following the $R$-peak with an amplitude of above $500 \mu \mathrm{V}$, a massive deflection between 150 and $350 \mathrm{~ms}$ was evident, as it is characteristic for inside scanner ECG recordings. Fig. 2A also shows the evoked BCG artefact scalp voltage map at selected latencies in BCG epochs. Note the variability of the artefact topography over the course of the 150 to $350 \mathrm{~ms}$ interval,
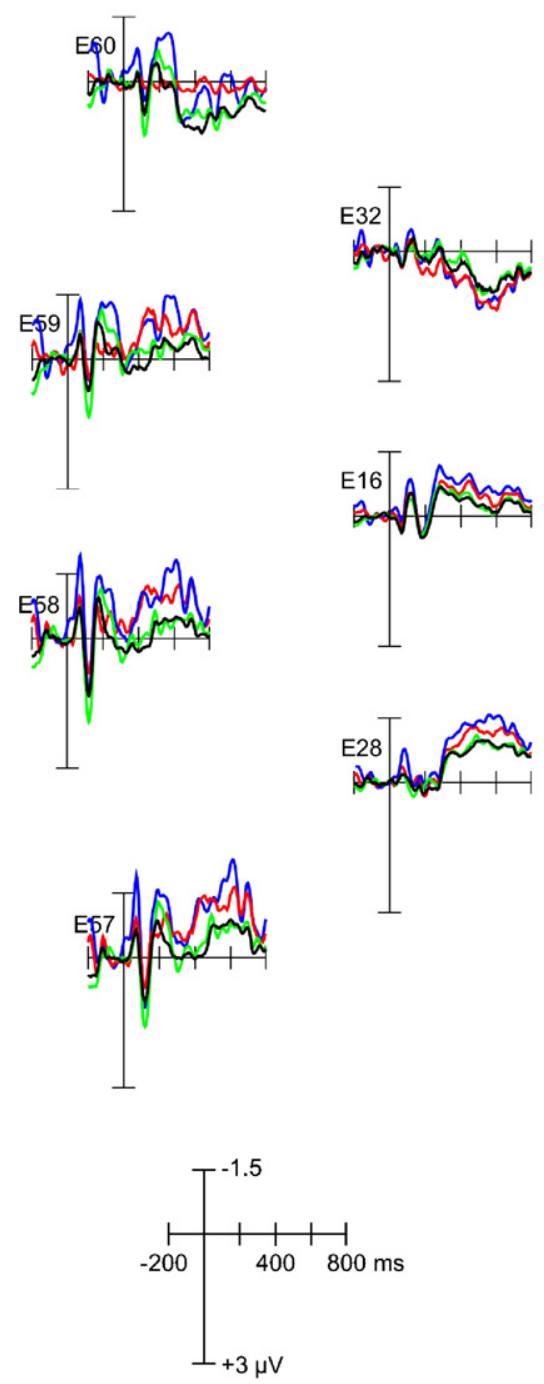
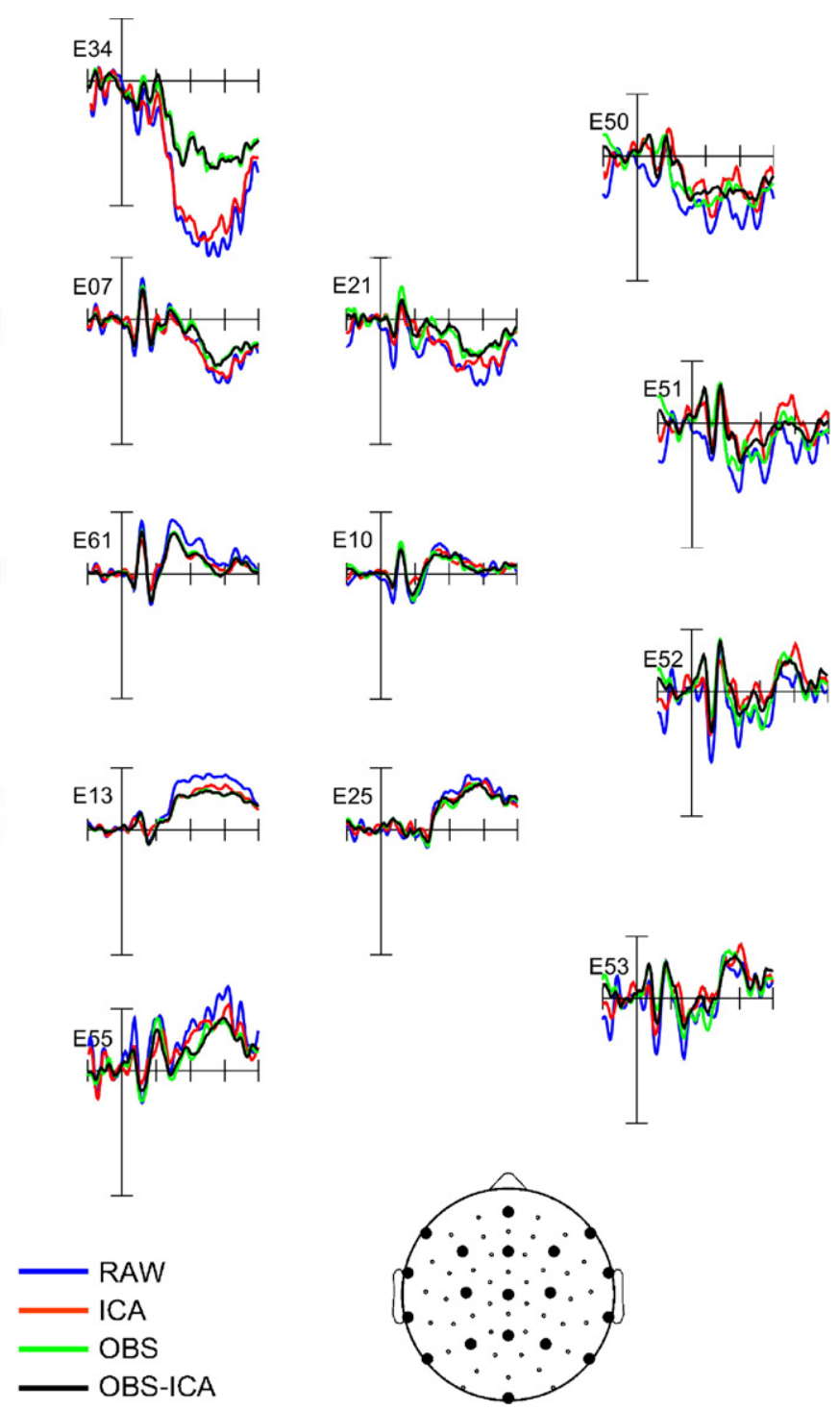

Fig. 3. Grand average auditory evoked potentials $(N=12)$ shown for a subset of 19 out of 60 EEG channels following different BCG correction procedures. Inset in the lower right corner of the figure indicates the layout of the selected channels. Channel with label E61 corresponds to vertex (Cz), other channels are similar to, but do not exactly overlap with the 10-20 electrode system. Further abbreviations as in Fig. 2. 
which also corresponds to the largest BCG artefacts on the unaveraged EEG channel level (see Fig. 1). Similar topographies were observed in all subjects. Fig. 2B shows the channel envelope for the evoked BCG artefact of the same subject before (RAW, blue) and after (ICA, red) BCG correction. The automatically identified IC maps for this subject are displayed as well, showing reasonable similarities with the voltage maps displayed in Fig. 2B. In this subject, the evoked BCG artefact was massively reduced by $59 \%$. A residual BCG artefact contribution of about $40 \mu \mathrm{V}$ was not accounted for, indicating a suboptimal correction. Fig. 2C shows the evoked BCG artefact of the same subject for the OBS and the OBS-ICA method. By applying the OBS method, the residual evoked BCG artefact was reduced to less than $1 \mu \mathrm{V}$. Furthermore, OBS-ICA identified one IC closely resembling the first IC shown in Fig. 2B. OBS-ICA further reduced the evoked BCG artefact by $32 \%$, leaving less than $0.5 \mu \mathrm{V}$ residual evoked BCG artefact in this subject.

Fig. 2D summarizes the statistical results for all subjects. A significant main effect $\left(F_{(1,11)}=74.65, p<.0001, \eta^{2}=0.872\right)$ confirmed that the data sets differed with regard to the amount of BCG artefact. As compared to the uncorrected RAW data, and indicated by the single subject demonstration (Fig. 2B), application of the ICA method significantly reduced $44 \%$ of the BCG artefact $\left(t_{11}=5.07, p<0.0004\right)$. A further massive reduction of the BCG artefact was evident when comparing OBS with ICA $\left(t_{11}=5.89, p<0.0002\right)$, indicating a mean residual BCG artefact of less than $1 \mu \mathrm{V}$. Albeit smaller in effect size, the OBS-ICA approach resulted in an additional and significant reduction of the residual BCG artefact when compared to OBS $\left(t_{11}=2.82\right.$, $p<0.05)$.

\section{Signal-to-noise ratio of AEPs}

Fig. 3 depicts the AEPs at 19 representative electrode locations (among them those displayed in Fig. 1) for the RAW data as well as the three different BCG correction procedures. Whereas the P1N1-P2 AEP complex was clearly evident in all datasets at frontocentral scalp sites, the amount of residual artefact at lateral channels was different. Note, for instance, the profound asymmetry between lateral left (E58) and right (E52) hemisphere channels in the RAW data at the interval of the P1-N1, which is not common for AEP recordings obtained outside the MR.
A

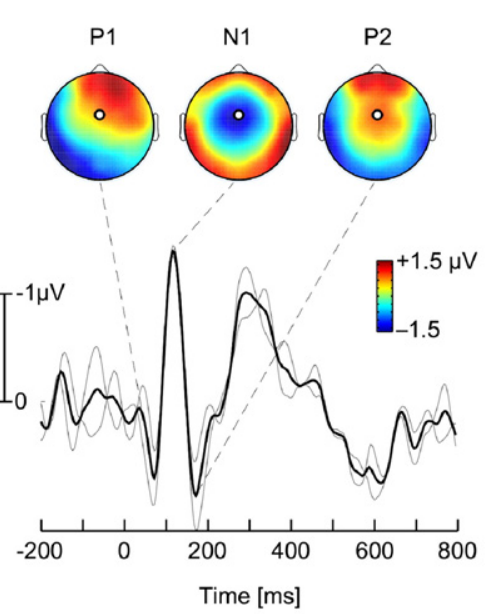

C

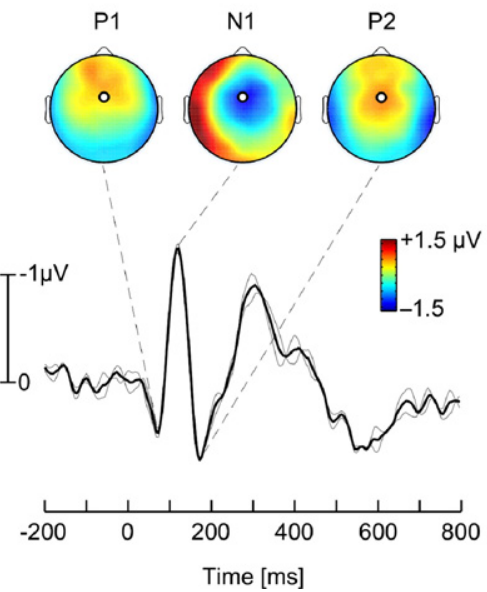

B

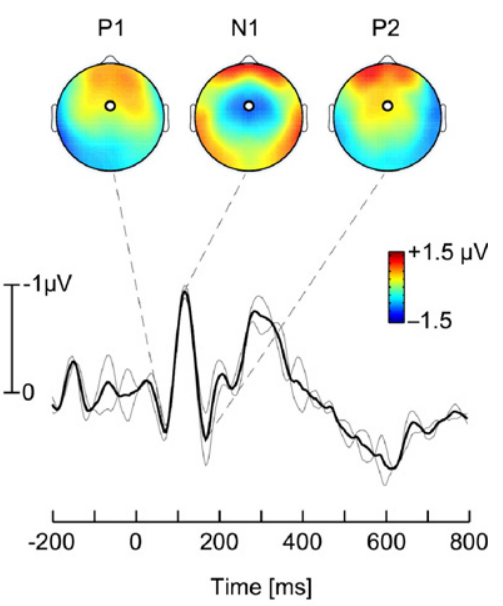

D

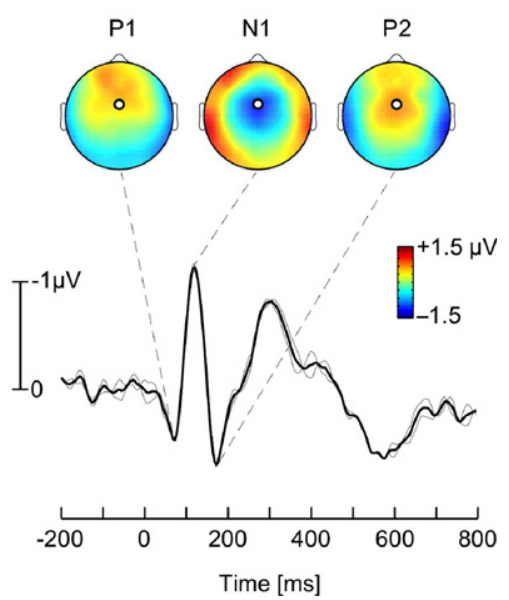

Fig. 4. Grand average auditory evoked potentials $(N=12)$ shown for a fronto-central channel, and corresponding voltage maps at $\mathrm{P} 1, \mathrm{~N} 1$, and $\mathrm{P} 2$ peak latencies. Thin lines show AEPs for odd and even trial averages. (A) AEPs for raw data uncorrected for the BCG artefact. (B) AEPs for BCG-corrected data using the ICA approach. (C) AEPs for BCG-corrected data using the OBS approach. (D) AEPs for BCG-corrected data using the combined OBS-ICA approach. 
Fig. 4 shows the morphology of the grand average AEP at a fronto-central channel, and the corresponding peak latency scalp maps for the P1, N1 and P2. For the uncorrected RAW data, the subaverages based on odd and even trials indicated a residual amount of noise, in particular at P1 and P2 latencies (Fig. 4A). However, the odd-even noise level at the peak latency of the N1 was remarkably small. Using the same scaling, AEPs and odd-even averages are shown for ICA (Fig. 4B), OBS (Fig. 4C), and OBS-ICA data (Fig. 4D). Whereas the topographies at P1, N1, and P2 latencies were relatively similar and expressed rather subtle differences, the SNR between these datasets was different. For the ICA-based AEP, a significant reduction in the N1 amplitude was observed when compared to the RAW data $\left(t_{11}=3.48, p<0.01\right)$. Accordingly, the SNR significantly dropped from 5.80 to 3.90 (Wilcoxon, $p<0.05$ ). When compared to the uncorrected RAW data, the OBS approach resulted in an improved SNR (8.16), which reached marginal significance (Wilcoxon, $p=0.06$ ). The largest SNR was obtained for the combined OBS-ICA approach (8.68), which approached significance when compared to the SNR of the uncorrected RAW data (Wilcoxon, $p=0.05$ ). This SNR improvement was evident despite a significant reduction of the N1 amplitude for the OBS-ICA compared to the RAW data $\left(t_{11}=3.88, p<0.01\right)$.

\section{Equivalent current dipole modelling}

Equivalent current dipole modelling of the uncorrected grand average RAW-AEPs resulted in a location at coordinates $(x, y, z)=$ $\pm 27.2,-15.9,-14.1$, corresponding to a Euclidean distance of $3.21 \mathrm{~cm}$ from the reference location in primary auditory cortex. Fitting the orientations of two bilateral dipoles seeded into the reference region resulted in a reasonable fit, as indicated by a residual variance of $7.42 \%$. However, as illustrated in Fig. 5, all three BCG correction procedures improved the N1 source modelling result. ICA-based AEPs were fitted at coordinates $(x, y, z)= \pm 35.8,-14.9,-13.3$, with a distance of $2.63 \mathrm{~cm}$ to the reference location, and a residual variance of $8.47 \%$. A further improvement was found for the OBS-based source modelling, which was located at coordinates $(x, y, z)= \pm 31.5,-15.7,-5.6$, $2.32 \mathrm{~cm}$ away from the reference location. The OBS-ICA dataset was modelled with a similar fit at a location $1.09 \mathrm{~cm}$ from the reference location $([x, y, z]= \pm 40.7,-15.5,2.6)$, indicating a further improvement of the AEP N1 topography. Dipole source analysis of the grand average data was followed by single subject AEP N1 source modelling, which is summarized in Fig. 6. Compared to the grand average results (11 to $32 \mathrm{~mm}$ ), single-subject modelling resulted in a substantially larger localization error (43 to $66 \mathrm{~mm})$, reflecting the poorer SNR for single-subject as compared to grand average AEP data. Nevertheless, the comparison between correction procedures revealed the same order of effects as found for grand average data, that is, the smallest localization error was found for OBS-ICA, followed by OBS and ICA. On a descriptive level, all correction procedures indicated an improvement in source localization accuracy compared to uncorrected RAW data. This effect was statistically significant for OBS-ICA $\left(t_{11}=2.31\right.$,

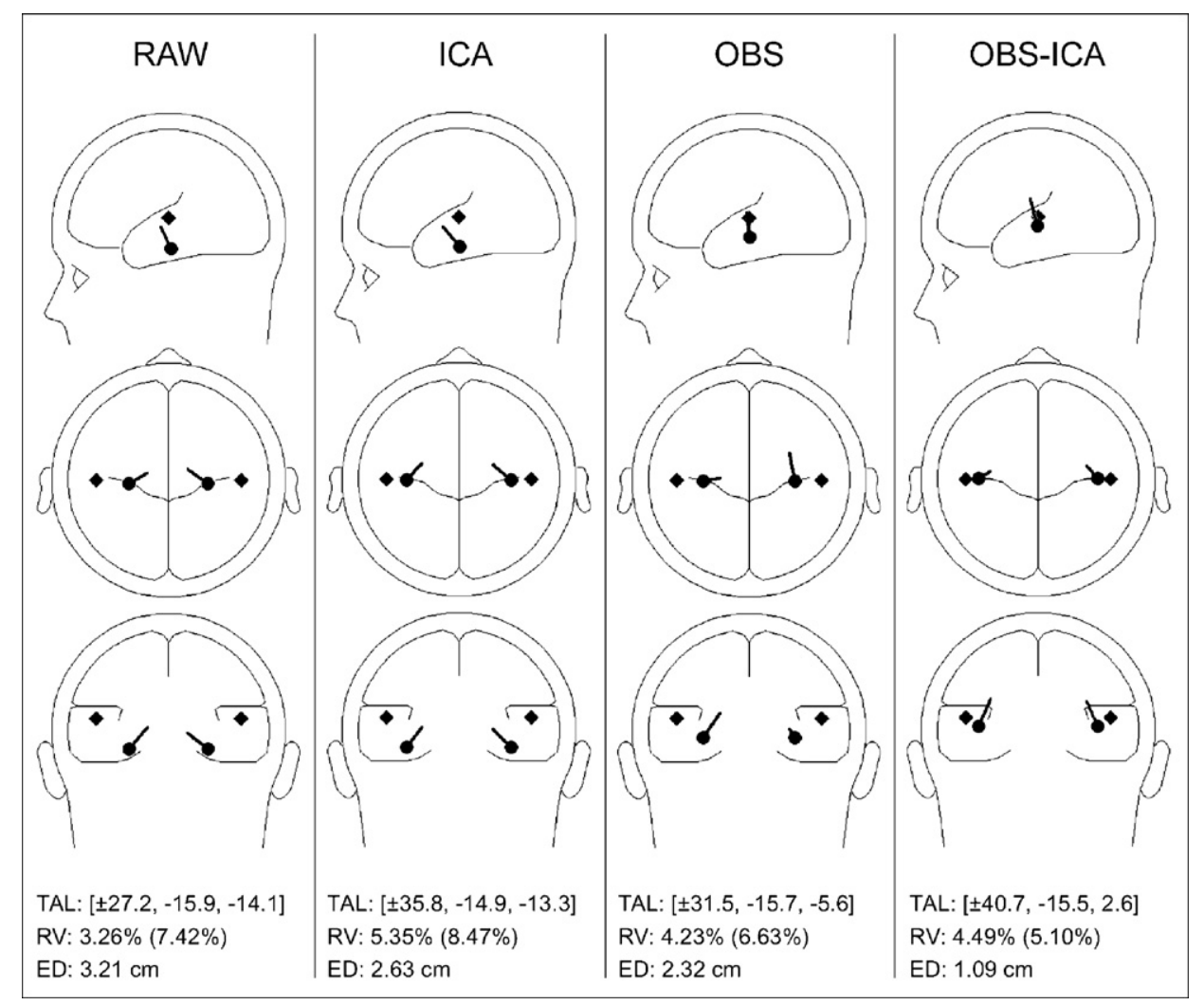

Fig. 5. Equivalent current dipole models of the grand average AEP N1 based on uncorrected (RAW) and three different BCG corrections of the same data. Black dipoles indicate the modelled location for the free model using a symmetry constraint. The corresponding Talairach coordinates (TAL) and residual variance (RV) values are given at the bottom. $R V$ in parenthesis refers to a seeded dipole model into the reference location (coordinates $\pm 49.5,-17,9$ ), which is indicated with black diamonds. $\mathrm{ED}=$ Euclidean distance. 


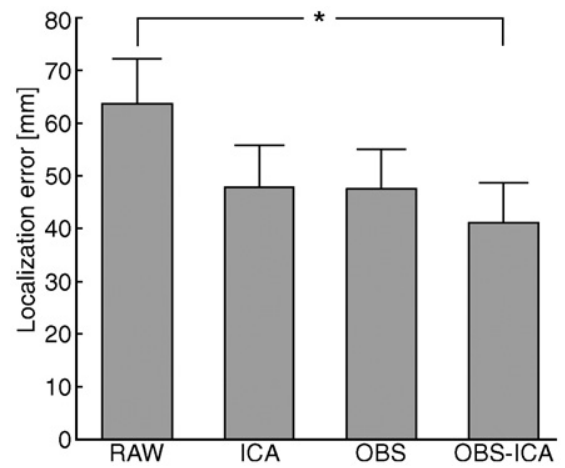

Fig. 6. Mean localization error (+SEM) for single-subject AEP N1 source localization. Localization error values refer to the Euclidean distance between modelled and reference locations. Abbreviations as in Fig. 2.

$p<0.05$ ), whereas ICA and OBS did not significantly reduce the localization error.

\section{Discussion}

To date, only a few studies have reported AEPs from EEG data recorded inside a scanner (Ellingson et al., 2004; Liebenthal et al., 2003; Mulert et al., 2005; Scarff et al., 2004). The present study adds to these reports by showing that the AEP N1 component can be recorded even with a TR as short as $2 \mathrm{~s}$. Moreover, we demonstrate that the choice of the BCG correction procedure has a profound influence on the quality of the AEP. By systematically comparing three recently proposed BCG correction methods, we found that, depending on the procedure applied, the SNR of the AEP N1 component can be significantly improved or reduced. Apparently, inappropriate BCG correction can make matters worse, whereas the application of an appropriate BCG correction strategy substantially improves the ERP quality.

A striking finding of the present study is the different performance of ICA, depending on whether it was used alone to account for the BCG artefact (Benar et al., 2003; Eichele et al., 2005; Srivastava et al., 2005), or whether it followed an OBSbased BCG correction procedure (Debener et al., 2005b). Compared to uncorrected data, the ICA approach resulted in a substantial BCG artefact reduction of about $50 \%$. This finding indicates that the correct ICs were identified by the correlation procedure applied (Srivastava et al., 2005), but it came at some expense. Both the N1 amplitude and the N1 SNR were reduced for ICA corrected data when compared to BCG-uncorrected data, rendering this procedure rather unsuited for the task at hand. In contrast, the application of ICA following OBS improved the resulting data quality. Even though the N1 amplitude was reduced, the SNR showed a significant increase of $43 \%$ when compared to uncorrected data. Importantly, the same pattern of results was also reflected in the topographical analysis of the AEP N1. Dipole seeding of the N1 ICA-AEP performed rather poorly, whereas modelling of the N1-AEP following OBS and OBS-ICA BCG correction gave rise to satisfactory results. Accordingly, usage of OBS-ICA for single-subject N1-AEP source localization significantly reduced the localization error.

The apparent difference in the performance of ICA can be explained by the nature of the BCG artefact contribution to the EEG signal. Over the period of each heartbeat cycle, the BCG artefact expresses a dynamically moving/rotating rather than a spatially fixed topography (for a movie illustrating this, see www. debener.de/eegfmri.html). Accordingly, a fixed spatial filter approach such as temporal ICA has difficulties decomposing this signal into one, or a few, components. For the same reason, ICA may perform suboptimally whenever the relative position of the recording sensors to the signal source(s) varies, for instance due to head motion in magnetoencephalogram (MEG) data, electrode cap movement in EEG, or a combined ICA across data from different subjects or sessions. The result of any shift in the relative position between source(s) and sensors is that the number of spatial patterns in a given dataset is inflated, while at the same time the number of dimensions that can be modelled remains constant. Due to the inflated spatial dimensionality in the data, maximizing temporal independence is harder to achieve. As a result, in the present study, ICA alone was not able to disentangle BCG-related activity from EEG-related activity. This conclusion is based on the observation that at the same time the N1 SNR and the amount of BCG artefact were reduced. The combined reduction of BCG artefact and N1 SNR argues against the possibility of identifying the wrong ICs. The procedure applied was fully automatic and motivated by a recent study (Srivastava et al., 2005) showing that several ICs correlated $(r>0.20)$ with the ECG signal. Although Srivastava et al. removed several additional components based on visual inspection of the corresponding IC activations, it can be ruled out that a similarly liberal component removal procedure could have led to a completely different result. While the removal of additional ICs may result in a larger suppression of the residual BCG artefact, it is unlikely to account for a reversal of the adverse effect on the N1 SNR. In addition, the visual inspection of IC activations (Benar et al., 2003; Srivastava et al., 2005) was complemented in the present study by an inspection of the IC maps, and confirmed that the automatic identification criterion applied was appropriate. Accordingly, the interpretation stands that ICA, when applied directly, does not perfectly separate EEG signal from BCG artefact contributions.

Regarding the OBS approach, the present results fit well with the original report (Niazy et al., 2005) and recent applications of OBS (Debener et al., 2005b; Iannetti et al., 2005). By removing more than $90 \%$ of the BCG artefact amplitude, the fully automatic OBS algorithm proved very useful. Importantly, OBS efficiently removed not only most of the BCG artefact, it also increased the SNR and reduced the source localization error of the AEP N1, although not to a significant extent. Compared to ICA, OBS corrected data could be modelled closer to the reference location in the Heschl gyrus, with a localization error of about $2 \mathrm{~cm}$ for grand average data, indicating a reasonable quality of the AEP N1 topography.

Several measures may be used to assess the quality of EEG data. The present study focused on the AEP N1 component and the results are likely to be valid for other ERP components as well. However, it should be noted that other quality measures, in particular in the frequency domain, could be applied as well. For instance, it would be interesting to know which BCG-removal strategy performs best to uncover well-known spectral EEG properties, like posterior alpha suppression for eyes open compared to eyes closed conditions. Given that the different BCG-removal strategies compared here may have removed a different amount of power from the data, we cannot fully exclude the possibility that the application of frequency-based measures may come to a slightly different result. Clearly, more research is needed to further 
improve the quality of EEG data recorded inside the scanner, and this research should focus on both event-related and spontaneous data.

In a recent study, we identified one IC after OBS-based BCG correction that reflected all major features of the error-related negativity, a cognitive ERP component (Debener et al., 2005b). This was not the case for the N1 component in the present study. However, a simple one-to-one relationship between ERP components and ICA components cannot generally be expected, since usually several ICs contribute to ERP components (for discussion, see Makeig et al., 2004a,b). While a thorough discussion of this issue is beyond the scope of this report, it should be noted that in the present study the application of ICA following OBS proved useful, since residual BCG-related artefact could be automatically identified and removed. As a result, the N1 SNR was significantly improved compared to uncorrected data, and the resulting grand mean AEP could be well modelled close to primary auditory cortex. Evidently, ICA performed well in separating residual BCG artefacts and brain-related EEG signals, because most of the moving/rotating part of the BCG artefact was already removed by OBS.

It might appear tedious to apply ICA following OBS, in particular when keeping in mind that in only one of the three quality measures applied, the difference between OBS and OBSICA was significant. In other words, OBS-ICA does not necessarily lead to a substantial improvement compared to OBS. However, the OBS-ICA approach as presented here was implemented in a fully automatic way and, on a descriptive level, resulted in the largest N1 SNR and smallest localization error. We therefore suggest that the combined application of OBS and ICA can be a powerful method for the analysis of EEG data collected simultaneously with $\mathrm{fMRI}$. This view goes along with our recent work suggesting that ICA, when applied after BCG-removal via OBS, can help to separate otherwise overlapping brain-related EEG signals and thus facilitates the integration of EEG and fMRI signals on a trial-by-trial level (Debener et al., 2005b; Debener et al., in press).

From its early days, equivalent current dipole seeding has been used for the integration of ERPs with neuroimaging data such as fMRI and positron emission tomography (e.g., Heinze et al., 1994). While fMRI-informed ERP dipole seeding may not solve the inverse problem, it may nevertheless provide information about the relationship between ERP and fMRI findings. By following this route, more recent accounts have established complex and detailed associations between fMRI and ERP signals obtained during cognitive tasks (Bledowski et al., 2004, 2006; Opitz et al., 1999). However, these studies combined ERP and fMRI signals obtained from separate recording sessions. By showing that simultaneous EEG/fMRI recordings can provide an ERP quality sufficient for ERP source modelling, we propose that future EEG/ fMRI integration work can profit from simultaneous recording protocols. Only the simultaneous acquisition of EEG and fMRI guarantees that both signals are recorded during identical sensory stimulation, subjective experience, and objective behaviour. Furthermore, many experimental designs investigating cognitive processes such as memory function and novelty processing are not well suited for repeated testing. And finally, a recently established temporal correlation approach now allows direct investigation of the possible coupling between event-related EEG and fMRI signals (Debener et al., 2005b; Eichele et al., 2005). This procedure requires not only the simultaneous recording of EEG and fMRI data but also a single-trial oriented EEG analysis approach (Debener et al., in press). By demonstrating systematic differences in the usage of ICA for the removal of the BCG artefact, the present study provides useful information for future research in this field.

\section{Acknowledgments}

We would like to thank Erik van den Bergh and Johan Gielissen for technical assistance, and Ingmar Gutberlet, Rami Niazy and Scott Makeig for accompanying discussions. This research was supported by a G.A. Lienert grant to A.S. and the NWO grant $402-$ 01-632 to J.P.

\section{References}

Allen, P.J., Polizzi, G., Krakow, K., Fish, D.R., Lemieux, L., 1998 Identification of EEG events in the MR scanner: the problem of pulse artifact and a method for its subtraction. NeuroImage 8 (3), 229-239.

Allen, P.J., Josephs, O., Turner, R., 2000. A method for removing imaging artifact from continuous EEG recorded during functional MRI. NeuroImage 12 (2), 230-239.

Bell, A.J., Sejnowski, T.J., 1995. An information-maximization approach to blind separation and blind deconvolution. Neural Comput. 7 (6), 1129-1159.

Benar, C.G., Aghakhani, Y., Wang, Y.H., Izenberg, A., Al-Asmi, A., Dubeau, F., Gotman, J., 2003. Quality of EEG in simultaneous EEGfMRI for epilepsy. Clin. Neurophysiol. 114 (3), 569-580.

Bledowski, C., Prvulovic, D., Hoechstetter, K., Scherg, M., Wibral, M., Goebel, R., Linden, D.E.J., 2004. Localizing P300 generators in visual target and distractor processing: a combined event-related potential and functional magnetic resonance imaging study. J. Neurosci. 24 (42), 9353-9360.

Bledowski, C., Kadosh, K.C., Wibral, M., Rahm, B., Bittner, R.A., Hoechstetter, K., Scherg, M., Maurer, K., Goebel, R., Linden, D.E.J., 2006. Mental chronometry of working memory retrieval: a combined functional magnetic resonance imaging and event-related potentials approach. J. Neurosci. 26 (3), 821-829.

Brechmann, A., Baumgart, F., Scheich, H., 2002. Sound-level-dependent representation of frequency modulations in human auditory cortex: a low-noise fMRI study. J. Neurophysiol. 87 (1), 423-433.

Debener, S., Kranczioch, C., Herrmann, C.S., Engel, A.K., 2002. Auditory novelty oddball allows reliable distinction of top-down and bottom-up processes of attention. Int. J. Psychophysiol. 46 (1), 77-84.

Debener, S., Herrmann, C.S., Kranczioch, C., Gembris, D., Engel, A.K., 2003. Top-down attentional processing enhances auditory evoked gamma band activity. NeuroReport 14 (5), 683-686.

Debener, S., Makeig, S., Delorme, A., Engel, A.K., 2005a. What is novel in the novelty oddball paradigm? Functional significance of the novelty P3 event-related potential as revealed by independent component analysis. Cogn. Brain Res. 22 (3), 309-321.

Debener, S., Ullsperger, M., Siegel, M., Fiehler, K., von Cramon, D.Y., Engel, A.K., 2005b. Trial-by-trial coupling of concurrent electroencephalogram and functional magnetic resonance imaging identifies the dynamics of performance monitoring. J. Neurosci. 25 (50), 11730-11737.

Debener, S., Ullsperger, M., Siegel, M., Engel, A.K., in press. Single-trial EEG-fMRI reveals the dynamics of cognitive function. Trends Cogn. Sci. [Electronic publication ahead of print; doi:10.1016/j.tics.2006. 09.010].

Delorme, A., Makeig, S., 2004. EEGLAB: an open source toolbox for analysis of single-trial EEG dynamics including independent component analysis. J. Neurosci. Methods 134 (1), 9-21.

Delorme, A., Makeig, S., Fabre-Thorpe, M., Sejnowski, T., 2002. From single-trial EEG to brain area dynamics. Neurocomputing 44, 1057-1064.

Eichele, T., Specht, K., Moosmann, M., Jongsma, M.L., Quiroga, R.Q., 
Nordby, H., Hugdahl, K., 2005. Assessing the spatiotemporal evolution of neuronal activation with single-trial event-related potentials and functional MRI. Proc. Natl. Acad. Sci. U. S. A. 102 (49), 17798-17803.

Ellingson, M.L., Liebenthal, E., Spanaki, M.V., Prieto, T.E., Binder, J.R., Ropella, K.M., 2004. Ballistocardiogram artifact reduction in the simultaneous acquisition of auditory ERPS and fMRI. NeuroImage 22 (4), 1534-1542.

Garreffa, G., Bianciardi, M., Hagberg, G.E., Macaluso, E., Marciani, M.G., Maraviglia, B., Abbafati, M., Carni, M., Bruni, I., Bianchi, L., 2004. Simultaneous EEG-fMRI acquisition: how far is it from being a standardized technique? Magn. Reson. Imaging 22 (10), 1445-1455.

Hall, D.A., Haggard, M.P., Akeroyd, M.A., Palmer, A.R., Summerfield, A.Q., Elliott, M.R., Gurney, E.M., Bowtell, R.W., 1999. "Sparse" temporal sampling in auditory fMRI. Hum. Brain Mapp. 7 (3), 213-223.

Hall, D.A., Summerfield, A.Q., Goncalves, M.S., Foster, J.R., Palmer, A.R., Bowtell, R.W., 2000. Time-course of the auditory BOLD response to scanner noise. Magn. Reson. Med. 43 (4), 601-606.

Heinze, H.J., Mangun, G.R., Burchert, W., Hinrichs, H., Scholz, M., Munte, T.F., Gos, A., Scherg, M., Johannes, S., Hundeshagen, H., Gazzaniga, M.S., Hillyard, S.A., 1994. Combined spatial and temporal imaging of brain activity during visual selective attention in humans. Nature 372 (6506), 543-546.

Iannetti, G.D., Niazy, R.K., Wise, R.G., Jezzard, P., Brooks, J.C., Zambreanu, L., Vennart, W., Matthews, P.M., Tracey, I., 2005. Simultaneous recording of laser-evoked brain potentials and continuous, high-field functional magnetic resonance imaging in humans. NeuroImage 28 (3), 708-719.

Jung, T.P., Makeig, S., Humphries, C., Lee, T.W., McKeown, M.J., Iragui, V., Sejnowski, T.J., 2000a. Removing electroencephalographic artifacts by blind source separation. Psychophysiology 37 (2), 163-178.

Jung, T.P., Makeig, S., Westerfield, M., Townsend, J., Courchesne, E., Sejnowski, T.J., 2000b. Removal of eye activity artifacts from visual event-related potentials in normal and clinical subjects. Clin. Neurophysiol. 111 (10), 1745-1758.

Kruggel, F., Wiggins, C.J., Herrmann, C.S., von Cramon, D.Y., 2000. Recording of the event-related potentials during functional MRI at 3.0 Tesla field strength. Magn. Reson. Med. 44 (2), 277-282.

Lee, T.W., Girolami, M., Sejnowski, T.J., 1999. Independent component analysis using an extended infomax algorithm for mixed subgaussian and supergaussian sources. Neural Comput. 11 (2), 417-441.

Liebenthal, E., Ellingson, M.L., Spanaki, M.V., Prieto, T.E., Ropella, K.M., Binder, J.R., 2003. Simultaneous ERP and fMRI of the auditory cortex in a passive oddball paradigm. NeuroImage 19 (4), 1395-1404.

Makeig, S., Debener, S., Onton, J., Delorme, A., 2004a. Mining eventrelated brain dynamics. Trends Cogn. Sci. 8 (5), 204-210.

Makeig, S., Delorme, A., Westerfield, M., Jung, T.P., Townsend, J., Courchesne, E., Sejnowski, T.J., 2004b. Electroencephalographic brain dynamics following manually responded visual targets. Plos Biol. 2 (6), $747-762$.

Mulert, C., Jager, L., Propp, S., Karch, S., Stormann, S., Pogarell, O., Moller, H.J., Juckel, G., Hegerl, U., 2005. Sound level dependence of the primary auditory cortex: simultaneous measurement with 61-channel EEG and fMRI. NeuroImage 28 (1), 49-58.

Naatanen, R., Picton, T., 1987. The N1 wave of the human electric and magnetic response to sound: a review and an analysis of the component structure. Psychophysiology 24 (4), 375-425.

Niazy, R.K., Beckmann, C.F., Iannetti, G.D., Brady, J.M., Smith, S.M., 2005. Removal of fMRI environment artifacts from EEG data using optimal basis sets. NeuroImage 28 (3), 720-737.

Onton, J., Delorme, A., Makeig, S., 2005. Frontal midline EEG dynamics during working memory. NeuroImage 27 (2), 341-356.

Opitz, B., Mecklinger, A., Friederici, A.D., von Cramon, D.Y., 1999. The functional neuroanatomy of novelty processing: integrating ERP and fMRI results. Cereb. Cortex 9 (4), 379-391.

Scarff, C.J., Reynolds, A., Goodyear, B.G., Ponton, C.W., Dort, J.C., Eggermont, J.J., 2004. Simultaneous 3-T fMRI and high-density recording of human auditory evoked potentials. NeuroImage 23 (3), $1129-1142$

Scherg, M., Von Cramon, D., 1985. Two bilateral sources of the late AEP as identified by a spatio-temporal dipole model. Electroencephalogr. Clin. Neurophysiol. 62 (1), 32-44.

Schicke, T., Muckli, L., Beer, A.L., Wibral, M., Singer, W., Goebel, R., Rösler, F., Röder, B., 2006. Tight covariation of BOLD signal changes and slow ERPs in the parietal cortex in a parametric spatial imagery task with haptic acquisition. Eur. J. Neurosci. 23, 1918-1920.

Schimmel, H., 1967. The $( \pm)$ reference: accuracy of estimated mean components in average response studies. Science 157 (784), 92-94.

Sijbers, J., Van Audekerke, J., Verhoye, M., Van der Linden, A., Van Dyck, D., 2000. Reduction of ECG and gradient related artifacts in simultaneously recorded human EEG/MRI data. Magn. Reson. Imaging 18 (7), 881-886.

Srivastava, G., Crottaz-Herbette, S., Lau, K.M., Glover, G.H., Menon, V., 2005. ICA-based procedures for removing ballistocardiogram artifacts from EEG data acquired in the MRI scanner. NeuroImage 24 (1), 50-60.

Walter, B., Blecker, C., Kirsch, P., Sammer, G., Schienle, A., Stark, R., Vaitl, D., 2003. MARINA: an easy to use tool for the creation of MAsks for Region of INterest Analyses (abstract). Presented at the 19th International Conference on Functional Mapping of the Human Brain, June 19-22, 2003, New York, NY. NeuroImage 19 (2) (Available on CD-ROM)

Yvert, B., Fischer, C., Bertrand, O., Pernier, J., 2005. Localization of human supratemporal auditory areas from intracerebral auditory evoked potentials using distributed source models. NeuroImage 28 (1), 140-153 